\title{
Cervical Approach of a Posterior Mediastinal Goiter: A Case Report
}

\author{
Althaf Ahmed ${ }^{1}$, Dhalapathy Sadacharan ${ }^{2}$, Smitha Sruti Rao ${ }^{3}$, PC Vijaykumar ${ }^{4}$
}

\begin{abstract}
Aim: To present a case of posterior mediastinal goiter successfully delivered through the neck approach.

Background: Posterior mediastinal goiters are rare, comprising less than $10 \%$ of intrathoracic goiters. They usually manifest with compressive symptoms and warrant definitive surgery to relieve symptoms. Majority require the combined cervicothoracic approach to deal with the goiter. Technique: We intend to discuss a case of retrosternal goiter extending predominantly into the posterior mediastinum, which has been purely delivered by cervical incision.

Conclusion: The above technique describes successful and safe extraction of the retrosternal goiter extending primarily into the posterior mediastinum via the cervical approach.

Clinical significance: The compressive symptoms were completely resolved after surgical management, thus avoiding a morbid procedure like thoracotomy and its associated complications.

Keywords: Airway compromise, Cervical approach, Compressive symptoms, Intrathoracic goiter, Posterior mediastinal extension.

World Journal of Endocrine Surgery (2019): 10.5005/jp-journals-10002-1269
\end{abstract}

\section{INTRODUCTION}

Intrathoracic goiters located anteriorly in the superior/anterior mediastinum are termed substernal or retrosternal goiters. Posterior mediastinal goiters are rare, comprising less than $10 \%$ of intrathoracic goiters. Large substernal nodular goiters can cause compression of surrounding structures, secondary hyperthyroidism, and malignant transformation. ${ }^{1-4}$ They may manifest with compression as well as displacement of mediastinal structures like trachea, esophagus, bronchi, large veins of the neck, and nerves. Clinical manifestations could be dyspnea, dysphagia, and hoarseness of voice. These become the major indications for surgery. Substernal goiter resections performed via the neck approach have less potential complications in comparison to invasive approaches like thoracotomy and combined cervicothoracic excision. They pose higher risk of intraoperative catastrophe, complications, as well as delayed postoperative recovery. ${ }^{5,6}$

\section{TechNique}

We present a case of a 78-year-old obese female who has known comorbidities of type 2 diabetes, systemic hypertension, toxic multinodular goitre on antithyroid medications since 4 years. She presented to the emergency room with increasing respiratory distress for 2 months and necessitated emergency endotracheal intubation. On further questioning, there was a history of progressive dysphagia. On further evaluation, chest X-ray showed mediastinal widening. Computed tomography (CT) of neck and chest was done and revealed $6.6 \times 5 \times 4.3 \mathrm{~cm}$ right lobe of thyroid with retrosternal extension. Another $9.1 \times 6.1 \times 5.5 \mathrm{~cm}$ multilobulated, heterogeneously enhancing lesion arising from left lobe of thyroid extending into posterior mediastinum involving the retrocarinal region with anterior displacement and compressing trachea, esophagus, major bronchi, superior vena cavae, ascending and descending thoracic aorta (Fig. 1). On biochemical evaluation, she was euthyroid. Upper Gl endoscopy revealed extraluminal compression of the esophagus at $15 \mathrm{~cm}$. In view of compressive symptoms, she was planned for emergency surgery to decompress the neck and mediastinum. \begin{tabular}{l}
\hline${ }^{1-3}$ Department of Endocrine Surgery, Madras Medical College, \\
Chennai, Tamil Nadu, India \\
${ }^{4}$ Department of Critical Care Medicine, Soorya Hospital, Chennai, Tamil \\
Nadu, India \\
Corresponding Author: Althaf Ahmed, Department of Endocrine
\end{tabular} Corresponding Author: Althaf Ahmed, Department of Endocrine
Surgery, Madras Medical College, Chennai, Tamil Nadu, India, Phone: +91 9790719570, e-mail: althafalways9@gmail.com

How to cite this article: Ahmed A, Dhalapathy S, Rao SS, et al. Cervical Approach of a Posterior Mediastinal Goiter: A Case Report. World J Endoc Surg 2019;11(3):100-102.

Source of support: Nil

Conflict of interest: None

She was planned for a combined cervical and posterolateral thoracotomy to remove the posterior mediastinal goiter. The patient was administered general anesthesia with a double-lumen endotracheal tube placed under guidance of a fiberoptic flexible bronchoscope. The Ryle's tube was inserted, and the central venous line was secured into the right subclavian vein. Then, the patient was positioned in a hyperextended position on a head ring with a shoulder bag behind the scapula to further extend the neck. Following skin preparation and draping, exposing the anterior neck up to the xiphisternum, $10 \mathrm{~cm}$ collar neck incision was placed two finger breadth above the sternal notch. Subplatysmal flaps were raised superiorly till the thyroid cartilage, inferiorly up to the clavicle. The lateral approach was chosen to lateralize sternomastoid muscles on both sides. The inferior belly of omohyoid was divided on both sides. Anterior jugular veins were ligated and divided. Strap muscles were divided on upper part both sides, and both lobes of thyroid were exposed. Initially, the right lobe of the thyroid was exposed, which showed multiple firm nodules with $4 \mathrm{~cm}$ retrosternal extension into the anterior mediastinum. The right superior pedicle was ligated, clamped, and divided. Right superior parathyroid glands were identified and preserved. The right recurrent laryngeal nerve was 

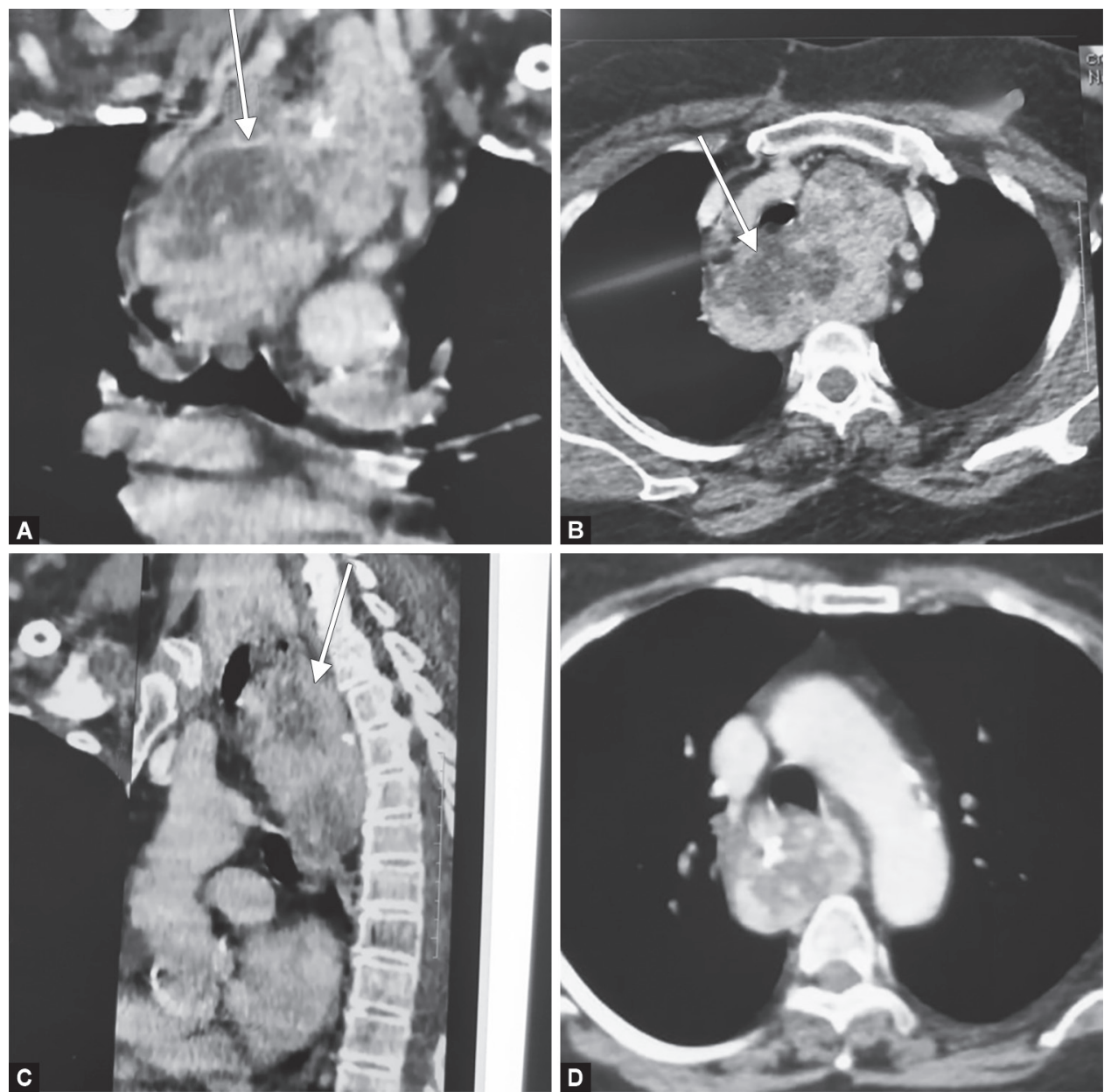

Figs $1 A$ to D: Computed tomography neck, chest: (A and B) Axial sections showing posterior mediastinal extension pushing the trachea ventrally; (C) Sagittal section showing the mediastinal goiter posterior to the trachea and esophagus; (D) Mediastinal goiter medial to the aortic arch

identified in its entirety till the cricothyroid space. The retrosternal extension of the right lower pole was delivered into the neck via the Toboggan technique staying anterior to the recurrent laryngeal nerve. The right inferior pedicle was ligated and divided. The right inferior parathyroid was identified and preserved. The right lobe was completely externalized. The left lobe of the thyroid had multiple firm nodules. It was associated with an intrathoracic extension into the posterior mediastinum for approximately $10 \mathrm{~cm}$ dipping retrotracheally, retroesophageally anterior to the thoracic vertebrae (Fig. 2). The left superior thyroid pedicle was ligated, clamped, and divided. The posterior mediastinal extension was delivered into the neck. Following this, the left recurrent laryngeal nerve was identified in the trachea-esophageal groove and exposed along its entire length up to the laryngeal entry point (Fig. 3). The left inferior thyroid pedicle was ligated and divided. The left inferior parathyroid was identified and preserved with the completion of total thyroidectomy. After attaining hemostasis, incision was closed in layers. The platysma was closed with a continuous absorbable suture and the skin closed with subcuticular absorbable sutures (Fig. 4). The postoperative period was uneventful with complete resolution of compressive symptoms and a normalization of patient's daily routine activities.

\section{Discussion}

Our patient had a large $9.1 \times 6.1 \times 5.5 \mathrm{~cm}$ multinodular goiter in the posterior mediastinum. An intrathoracic goiter represents

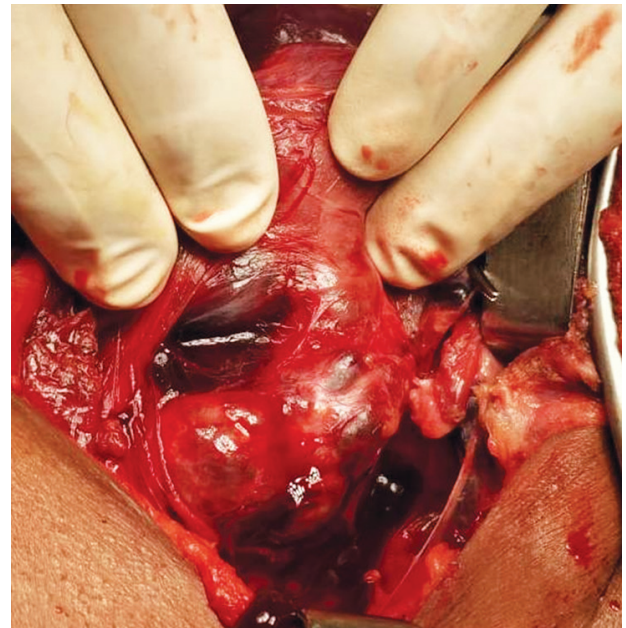

Fig. 2: Intraoperative picture of the posterior mediastinal goiter before delivery into the neck

downward extension of the cervical thyroid tissue into the thoracic cavity through the thoracic inlet. With progressive enlargement of the goiter, the path of least resistance is inferior into the thorax. ${ }^{7-9}$ The huge mass and weight of the goiter in our case affected the swallowing and respiration. The literature describes that the weight of the goiter with normal swallowing and respiratory motion favors 


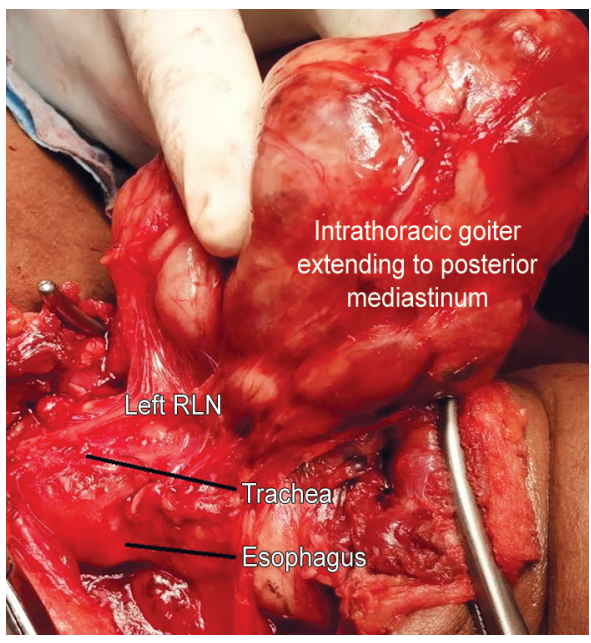

Fig. 3: Intraoperative picture postdelivery of the intrathoracic goiter

mediastinal displacement. ${ }^{5,9}$ Incidence of retrosternal goiters in general population is 1:5,000 and is seen in 5-16\% thyroidectomy specimens. The posterior mediastinal goiter is rare comprising $10 \%$ intrathoracic goiters. This necessitated the reporting of the detailed management in our case. ${ }^{2,5}$ As documented in our patient, these goiters are initially asymptomatic and later develop obstructive symptoms, signs due to compression, displacement of trachea, bronchi, esophagus, or large veins in the neck. ${ }^{10}$ Posterior mediastinal goiters should be differentiated from other mediastinal masses on appropriate workup. Computerized tomography of the neck with contrast delineated a large posterior mediastinal extension in our case. ${ }^{3,9}$ The surgical selective approach for excision of posterior mediastinal goiters is recommended for symptomatic obstructive goiters. We decided to approach our case cervically with the help of thoracic surgeons, if necessary. We could successfully extract the mediastinal extensions via the cervical approach with no requirement of thoracotomy. Khayat et al. have reported a complete cervical excision of the posterior mediastinal extension of a retrosternal goiter, which prevented the routine complications of thoracotomy. ${ }^{10}$ Posterior mediastinal goiters are preferred to be excised via a combined approach rather than purely cervical approaches due to their larger sizes and surrounding vital structures. Selective approach: thoracotomy, combined cervicothoracic approach is advocated for larger lesions, when mass is intrathoracic, no cervical component, or when mass is malignant. 11,12 The successful cervical excision is the highlight in this case, avoiding the significant morbidity of combined approaches/thoracotomies. Thoracotomy is associated with severe postoperative pain, infection, basal lung collapse, and respiratory failure, which had been totally avoided in our patient.

\section{CONCLUSION}

The posterior mediastinal goiter with mediastinal compressive symptoms is a definitive indication for surgery. Posterolateral thoracotomy is the main approach for intrathoracic extension into the posterior mediastinum. In this case, the patient had both types of retrosternal extension: predominantly into the posterior mediastinum, which was successfully delivered through the neck incision. Surgery was completed through cervical incision without thoracotomy, thus avoiding morbid complications. It is advisable to consider surgical management using a selective targeted approach

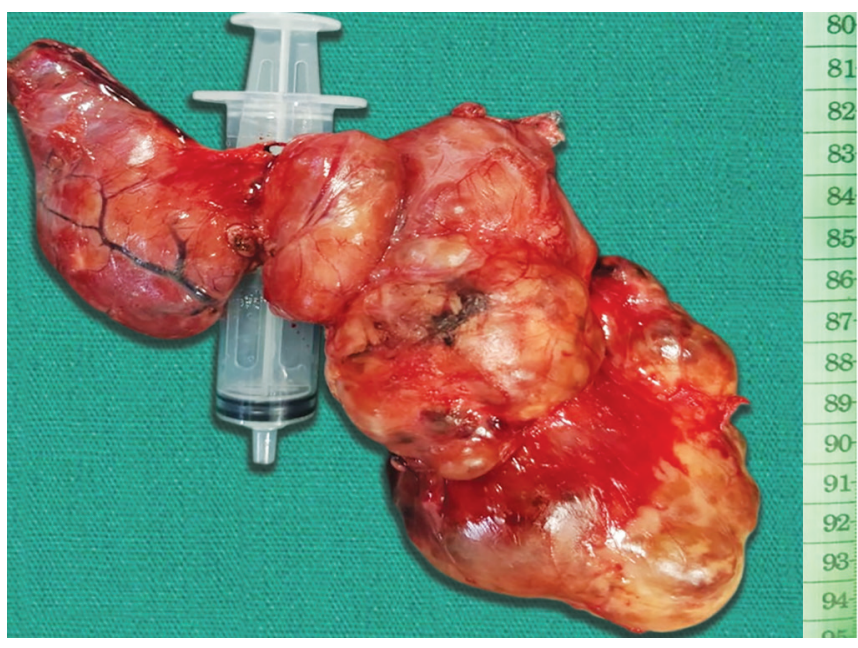

Fig. 4: Postoperative specimen

for the symptomatic mediastinal goiter with minimum morbidity and postoperative complications.

\section{References}

1. Ojanguren Arranz A, Baena Fustegueras JA, Ros López S, et al. Best approach for posterior mediastinal goiter removal: transcervical incision and lateral thoracotomy. Arch Bronconeumol 2014;50(6): 255-257. DOI: 10.1016/j.arbres.2013.09.009.

2. Gao $B$, Jiang $Y$, Zhang $X$, et al. Surgical treatment of large substernal thyroid goiter: analysis of 12 patients. Int J Clin Exp Med 2013;6(7):488-496.

3. Chen X, Xu H, Ni Y, et al. Complete excision of a giant thyroid goiter in posterior mediastinum. J Cardiothorac Surg 2013;8:207-209. DOI: 10.1186/1749-8090-8-207.

4. Allo MD, Thompson NW. Rationale for the operative management of substernal goiters. Surgery 1983;94(6):969-977.

5. McKenzie G, Rook W. Is it possible to predict the need for sternotomy in patients undergoing thyroidectomy with retrosternal extension? Interact Cardiovasc Thorac Surg 2014;19(1):139-143. DOI: 10.1093/ icvts/ivu094.

6. Fujimoto $\mathrm{K}$, Hara M, Tomiyama N, et al. Proposal for a new mediastinal compartment classification of transverse plane images according to the Japanese Association for Research on the Thymus (JART) General Rules for the Study of Mediastinal Tumors. Oncol Rep 2014;31(2): 565-572. DOI: 10.3892/or.2013.2904.

7. De Andrade MD. A review of 128 cases of posterior mediastinal goiter. World J Surg 1977;1(6):789-797. DOI: 10.1007/BF01555944.

8. Qureishi A, Garas G, Tolley N, et al. Can pre-operative computed tomography predict the need for a thoracic approach for removal of retrosternal goiter? Int J Surg 2013;11(3):203-208. DOI: 10.1016/ j.ijsu.2013.01.006.

9. Kacprzak G, Karas J, Rzechonek A, et al. Retrosternal goiter located in the mediastinum: surgical approach and operative difficulties. Interact Cardiovasc Thorac Surg 2012;15(5):935-937. DOI: 10.1093/ icvts/ivs339.

10. Khayat S, Al-Juaid A, Khorchid A, et al. Retrosternal goiter with posterior mediastinal extension surgery in a patient with superior vena cava obstruction through a classic neck incision: a case report. WebmedCentral Surgery 2015;6(5):WMC004890.

11. Dhaliwal RS, Puri D, Rana SS. Posterior mediastinal goiters: literature review and report of three cases. Asian Cardio Vasc Thorac Ann 1999;7:228-232. DOI: 10.1177/021849239900700315.

12. Samokhvalova A, Loberantb N, Makhoula N. Posterior mediastinal goiters: report of two cases and literature review. Respir Med Case Rep 2012;5:65-68. DOI: 10.1016/j.rmedc.2011.08.001. 